\title{
Neonatal Herpesvirus hominis infections
}

\author{
R. W. JOHN \\ J. O'H. TOBIN \\ University Hospital of South Manchester and Public Health Laboratory, Withington Hospital, \\ Manchester M20 8LR
}

Herpesvirus hominis (HVH) infections of the newborn have been documented since 1934 (Batignani, 1934), but only in recent years has the full range of clinical presentation become apparent and treatment become a possibility. A recent review of their experiences with this disease by Nahmias, Alford \& Korones (1970) has shown that in North America the disease tends to follow three patterns: a disseminated form with a high mortality (over $80 \%$ ), a localized form with severe mortality $(44 \%)$ if involving the central nervous system, but not otherwise, and the very occasional case of asymptomatic infection.

Four cases of neonatal HVH infection which have been diagnosed in Manchester during the last 2 years are described here. The clinical features are summarized in the table.

TABLe 1. Clinical features

\begin{tabular}{lcc}
\hline & $\begin{array}{c}\text { Overall \& } \\
\text { Glasgow, 1970 }\end{array}$ & Present series \\
\hline No. of cases & 43 & 4 \\
Symptoms and signs (\%) & & \\
Respiratory & 60 & 75 \\
Feeding difficulty & 58 & 75 \\
Skin lesions & 53 & 75 \\
Abnormal temperature & 51 & 75 \\
Hepatosplenomegaly & 51 & 50 \\
Bleeding & 13 & 50 \\
Keratitis/conjunctivitis & 30 & 25 \\
Outcome (\%) & & \\
Died & 69 & 50 \\
\hline
\end{tabular}

\section{Case histories}

\section{Case 1. Male infant K.H.}

This baby was born on 20 March 1971 at 40 weeks' gestation, birth weight $3.629 \mathrm{~kg}$, the second pregnancy of a healthy mother. Onset of labour was spontaneous and a normal vertex delivery ensued. The baby was normal on examination and was discharged home at $48 \mathrm{hr}$.

At 4 days of age he began coughing, had a hoarse cry and the following day developed mild respiratory symptoms and fed poorly. On the ninth day he was admitted to hospital mainly because a routine throat swab taken whilst in hospital had grown HVH type 1. He was febrile $\left(39.6^{\circ} \mathrm{C}\right)$, dyspnoeic and had a palpable liver $(2.5 \mathrm{~cm})$ and spleen tip. One pink macule was present on his face. No neurological abnormality was noted. The initial $\mathrm{WBC}$ was $10,600 / \mathrm{mm}^{3}(\mathrm{~N} 58 \%$, $\mathrm{L} 36 \%$, E $4 \%$, M $3 \%$ ) and a chest X-ray showed inflammatory changes at the right base and right middle lobe.

He was treated with cytarabine $5 \mathrm{mg} / \mathrm{kg}$ body weight intravenously for 5 daily doses and given intramuscularly pooled immunoglobulin $(750 \mathrm{mg})$ on the ninth and fifteenth day of life. He showed initial clinical improvement but on the eleventh day developed conjunctivitis, on the eighteenth vomited a small amount of blood and on the twenty-fourth day required sedation because of agitation. He then improved and was discharged home at 34 days of age. When last seen 8 months later he appeared a normal infant with normal developmental attainments.

Investigations. Bacteriological cultures of throat swab and blood were negative and subsequent blood counts normal. After admission HVH type 1 was cultured from throat swabs on the first 4 days of treatment, but not subsequently, and his herpes complement-fixing antibodies rose from 1:8 on day 10 to $1: 256$ on day 15. Specific fluorescence for $\mathrm{HVH}$ was shown by Dr Maurice Longson in nasal aspirate taken on the day of hospital admission.

This infant's mother was admitted to hospital 1 month postpartum with a febrile illness, sore throat, headache and aches in the legs. She became asymptomatic rapidly and bacterial and viral cultures were negative. Her serum herpes simplex antibody titre was $1: 128$ at this time, but as the serum was taken on the second day of her symptoms these cannot have been due to HVH infection. A comparison of the complement-fixation titres in infant and mother would indicate perinatal infection in the latter.

\section{Case 2. Male infant $\boldsymbol{H}$.}

This baby was born on 1 July 1971 at 39 weeks' gestation, birth weight $3.203 \mathrm{~kg}$, by vaginal delivery following pitocin drip induction. For 3 weeks before delivery his mother had noted some soreness of the 
genitalia. On initial examination the baby was normal, but at 3 days he became febrile $\left(38 \cdot 8^{\circ} \mathrm{C}\right)$. On the fifth day of life he was tachypnoeic and sucking poorly and a blister appeared on his head. He was started on cytarabine treatment, $5 \mathrm{mg} / \mathrm{kg}$ body weight intravenously daily, for 5 days. Throat swabs taken at this time yielded $\mathrm{HVH}$ type 2 . He remained ill and by the eighth day had developed purpura. At this time his platelet count was normal but subsequently fell to $40,000 / \mathrm{mm}^{3}$ and umbilical bleeding occurred. Haematological investigations excluded disseminated intravascular coagulation. On the thirteenth day he developed new vesicles on his back which were treated with local idoxuridine. His respiratory symptoms continued despite intensive antibiotic therapy and he collapsed and died aged 16 days. Pooled immunoglobulin $(1500 \mathrm{mg})$ was not given until the tenth day of life. A chest X-ray on the ninth day had shown widespread bronchopneumonic consolidation.

Necropsy showed areas of necrosis in the liver, lungs and adrenals but no inclusion bodies were seen. HVH was cultured from the trachea and liver. A bacterial pneumonia was found and lung cultures yielded antibiotic-resistant coliform bacilli. The brain appeared normal.

His mother was shown by virus isolation to have genital herpes which was treated with local idoxuridine.

Fluorescent antibody tests (IgG) on a serum sample taken on 5 July 1971 from this infant had a titre of $1: 56$ and on 6 July 1971 from the mother a titre of $1: 1458$. These titres indicate perinatal infection in the mother.

\section{Case 3. Male infant J.H.}

This baby was born on 25 August 1969 at 40 weeks' gestation, birth weight $3.515 \mathrm{~kg}$, following a normal pregnancy. At 1 day of age he became tachypnoeic and pale but the next day he was better clinically and was discharged home at 9 days. HVH was isolated from a routine urine specimen taken on day 8, but he received no treatment for this. Aged 1 month he was admitted to hospital with pyloric stenosis and this was treated surgically with no complications. At this time no HVH was isolated from the baby and on examination he was normal. $\mathrm{He}$ has subsequently failed to attend follow-up appointments.

\section{Case 4. Female infant R.F.}

This baby was born on 1 August 1969 at 40 weeks' gestation, birth weight $2.570 \mathrm{~kg}$ (less than fifth percentile) following a normal pregnancy and labour. At 8 days of age she became febrile $\left(38.8^{\circ} \mathrm{C}\right)$ and was irritable. Antibiotic treatment was started but she remained febrile and unwell despite a change of antibiotics. At 12 days of age skin vesicles appeared and on the fourteenth day the liver became enlarged and she bled from her mouth and buttocks. She was treated with vitamin $\mathrm{K}$, hydrocortisone and fresh blood was transfused, but she died on the fourteenth day.

Investigations. The haemoglobin fell from $15 \mathrm{~g}$ to $10.8 \mathrm{~g}$ on the last day of life. WBC count was $12,600 /$ $\mathrm{mm}^{3}(\mathrm{~N} 75 \%$, L $11 \%$, M $11 \%$, E 3\%). Bacteriological cultures were negative. On day 14 the prothrombin time was unrecordable and platelet count was $28,000 / \mathrm{mm}^{3}$. A throat swab from the tenth day grew HVH type 1. No herpes antibodies were present in maternal serum 16 days postpartum.

Necropsy examination showed an enlarged liver with petechiae over its surface. Necrotic areas and intranuclear inclusion bodies were seen on histological examination. The lungs showed areas of congestion and collapse with large mononuclear cells present.

\section{Discussion}

The four infants described here illustrate the variability of clinical features found with HVH infections. Three of the infants suffered from the disseminated type of disease and two of these died, the survivor being one of two treated with cytarabine. The fourth infant had mild, transient respiratory symptoms.

No definite signs of CNS involvement were noted either clinically or at necropsy although Nahmias et al. (1970) report CNS abnormalities in forty out of ninety-eight infants with disseminated $\mathrm{HVH}$ infections. Case 4 may have illustrated the development of disseminated intravascular coagulation or a bleeding diathesis due to necrosis of the liver. $40 \%$ of fatal disseminated HVH infections of the newborn are said to be associated with bleeding (Miller et al., 1970). The appearance of skin vesicles makes the diagnosis more obvious but in none of these infants were skin vesicles present at the start of the illness. Music, Fine \& Togo (1971) reported a case in which the vesicles occurred in a zoster-like distribution.

Cytarabine (cytosine arabinoside) has previously been used for the treatment of varicella zoster infections (Prager, Bruder \& Sawitsky, 1971), for herpes simplex keratitis (Kaufman \& Maloney, 1963) and generalized HVH infections (Juel-Jensen, 1970; Rappel, 1971). Of the two babies treated with cytarabine one (Case 1) recovered with no apparent sequelae being demonstrated at 9 months of age. The other treated baby died at 16 days but had a superadded bacterial pneumonia. Although no inclusion bodies were seen at necropsy in this treated case, HVH was isolated from the trachea and liver. This is in contrast to the case who died following no chemotherapy, who showed many inclusion bodies in the organs at 
necropsy. There were two differences in the situations of the babies treated with cytarabine in that the one who recovered was infected by a type $1 \mathrm{HVH}$ and had immunoglobulin on the first day of treatment, while the second was infected by a type 2 virus and received immunoglobulin on the fifth day of treatment. Immunoglobulin by itself has been shown to be ineffective even if given before symptoms develop (Nahmias et al., 1970). However, combined with cytarabine, which markedly reduces the amount of virus but does not eliminate it from the patient, it is possible that it might have some cumulative effect. Rather crude sensitivity tests in cell culture with the strains from these two patients showed that both were sensitive to $1 \mu \mathrm{g}$ of cytarabine per $\mathrm{ml}$ of maintenance medium. Clinically both cases were very similar initially and treatment was started early in both illnesses. Both were diagnosed early; in Case 2 the mother had genital herpes and in Case 1 the virus was isolated from a routine swab taken on the second day of life, the baby being admitted to hospital as soon as the virus was identified and before a definite clinical diagnosis of his symptoms had been made.

The infecting virus as judged by the production of pocks on the chorioallantoic membrane of the developing chick embryo was type 1 in two cases and by more sophisticated methods (Longson, 1971), one other was shown to be type 1 and the other type 2 . This predominance of type $1 \mathrm{HVH}$ contrasts with the findings of Nahmias et al. (1970) who considered that type 2 was the predominant strain in their neonatal infections. In Finland, type 2 also seems to be the common causal agent (Pettay et al., 1972). In Cases 1 and 2, infection was derived perinatally from the mother, while in Case 4 someone else was presumably the source of infection, as HVH antibody was not detected in the mother's blood 16 days after delivery. The source of infection in Case 3 was not determined.

The overall incidence of neonatal HVH infections in North America has been estimated at one clinically recognized case per 7500 deliveries, but with a frequency varying from $1 / 3500$ to $1 / 30,000$ deliveries (Nahmias et al., 1970). Two of our cases were born in one hospital, which in that establishment would give an incidence of one case per 4860 live births. Genital herpes is very rarely seen amongst hospital patients in this area, being confined almost entirely to patients attending the venereal disease clinics.

Because of the unpredictable nature of $\mathrm{HVH}$ infections in the newborn no conclusions can be drawn about the effectiveness of cytarabine therapy from the recovery with no sequelae in one of two cases treated. However, there is no doubt that this baby had a disseminated infection with a pneumonitis and hepatosplenomegaly and in the series of $\bar{z}$ Nahmias et al. (1970) the chances of survival with $\varnothing$ such disease would be low. In two cases treated with $c$. idoxuridine by Pettay et al. (1972) one recovered $\vec{F}$ completely, the other, together with seven other $\frac{\text { ? }}{?}$

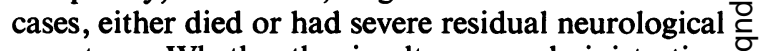
symptoms. Whether the simultaneous administration $\frac{\bar{c}}{\bar{c}}$. of immunoglobulin helped in our case that recovered $\frac{\sigma}{\sigma}$ has also to be considered, but it probably should be $\varnothing$ given in all cases irrespective of its effectiveness, as $ळ$ recommended by the American workers.

The diagnosis of neonatal herpes infection can $\stackrel{-}{-}$ obviously be very difficult, especially if no skin lesions $\vec{\omega}$ are visible to give some indication of the infection. $\frac{\text { of }}{0}$ In our case that recovered no obvious skin lesions appeared until after treatment of the patient had $\stackrel{3}{*}$. started, by which time it is possible that treatment $\frac{0}{0}$ would not have been effective. The cytarabine had of little effect on the blood cells in one case, but in the $N$ other who died there was marked lowering of the $\mathscr{\omega}_{0}$ platelet count.

\section{Acknowledgments}

Our thanks are due to Dr G. V. Feldman, Dr D. Macaulay and Dr R. I. Mackay for permission to publish their cases; to $\mathbb{D}$ Dr M. Longson for help in typing the viruses isolated and $\overrightarrow{0}$ advice on treatment with cytarabine; to Dr S. Whittaker ang Dr R. Gillett for necropsy reports.

\section{References}

Batignani, A. (1934) Congiuntivite da virus erpetico in neonato. Bolletino d'oculistica, 13, 1217.

JUEL-JENSEN, B. E. (1970) Severe generalised primary herpes treated with cytarabine. British Medical Journal, 2, 154.

Kaufman, H. E. \& Maloney, E. D. (1963) IDU and cytosine $\overrightarrow{\bar{o}}$ arabinoside in experimental herpetic keratitis. Archives of 3 Ophthalmology, 69, 626.

LoNGSON, M. (1971) A temperature marker test for the differentiation of strains of Herpesvirus hominis. Annales de l'Institut Pasteur, 120, 699.

Miller, D. R., Hanshaw, J. B., O'Leary, D. S. \& Hnilicka, J. V. (1970) Fatal disseminated herpes simplex virus $\bar{\sigma}$ infection and hemorrhage in the neonate. Journal of 3 . Pediatrics, 76, 409.

Music, S. I., FIne, E. M. \& Togo, Y. (1971) Zoster-like disease in the newborn due to herpes-simplex virus. New England Journal of Medicine, 284, 24.

Nahmias, A. J., Alford, C. A. \& Korones, S. B. (1970) Infection of the newborn with Herpesvirus hominis. Advances in Pediat rics, 17, 185.

Overall, J. C. \& Glasgow, L. A. (1970) Virus infections of N the fetus and newborn infant. Journal of Pediatrics, 77, $\sigma$ 315.

Pettay, O., Leinikei, P., Donner, M. \& Lapinleimu, K. N (1972) Herpes simplex virus infection in the newborn. $\omega$ Archives of Disease in Childhood, 47, 97.

Prager, D., Bruder, M. \& Sawitsky, A. (1971) Dissemi- 0 nated varicella in a patient with acute myelogenous $\frac{\bar{D}}{\Phi}$ leukaemia: treatment with cytosine arabinoside. Journal \& of Pediatrics, 78, 321.

RAPPEL, M. (1971) Treatment of herpesvirus encephalitis. Lancet, i, 971. 